
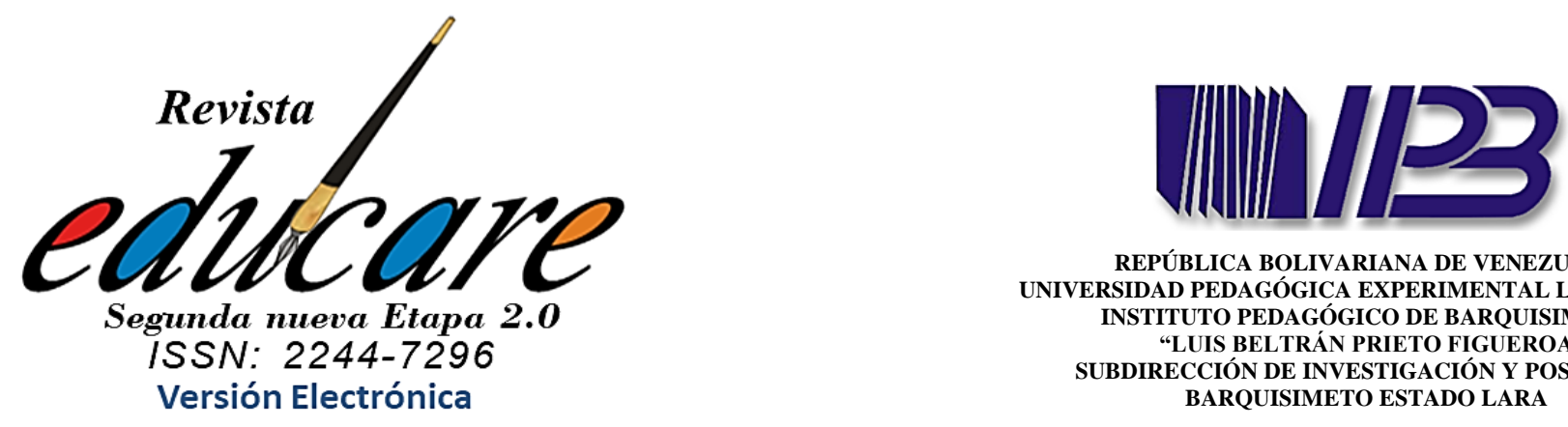

REPÚBLICA BOLIVARIANA DE VENEZUELA UNIVERSIDAD PEDAGÓGICA EXPERIMENTAL LIBERTADOR INSTITUTO PEDAGÓGICO DE BARQUISIMETO "LUIS BELTRÁN PRIETO FIGUEROA

SUBDIRECCIÓN DE INVESTIGACIÓN Y POSGRADO BARQUISIMETO ESTADO LARA

Volumen 24 № 1 Enero-Abril 2020

$(222-244)$

Desirée Margarita Martinéz Siejas

ORCID: https://orcidorg/0000-0002-2887-2279

\author{
UNIVERSIDAD PEDAGÓGICA \\ EXPERIMENTAL LIBERTADOR \\ VENEZUELA
}

\section{LA MEDIACIÓN COMO ESTRATEGIA DE RESOLUCIÓN DE CONFLICTOS PACÍFICA EN EL ÁMBITO ESCOLAR}

MEDIATION AS A PACIFIC CONFLICT RESOLUTION STRATEGY IN THE SCHOOL SCOPE

Profesora en Educación. Doctorante en Ciencias de la Educación-Convenio Universidad Pedagógica Experimental Libertador-Colegio Universitario de Administración y Mercadeo. Desireemartinez16@hotmail.com

Recibido:

27-06-2019

Aceptado:

16-01-?חวก 

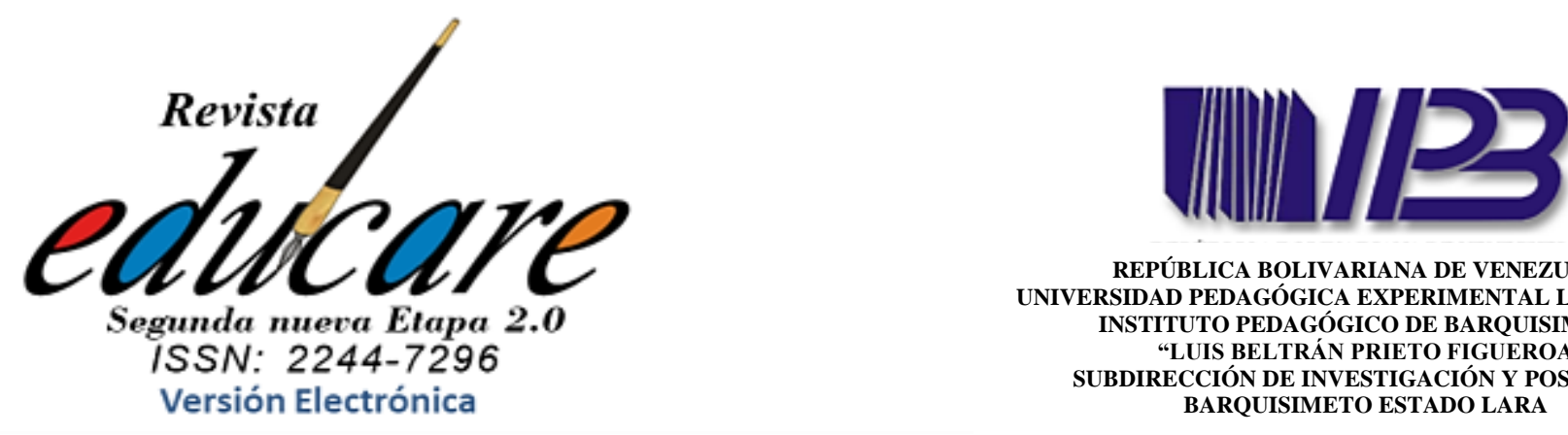

REPÚBLICA BOLIVARIANA DE VENEZUELA UNIVERSIDAD PEDAGÓGICA EXPERIMENTAL LIBERTADOR INSTITUTO PEDAGÓGICO DE BARQUISIMETO "LUIS BELTRÁN PRIETO FIGUEROA SUBDIRECCIÓN DE INVESTIGACIÓN Y POSGRADO BARQUISIMETO ESTADO LARA

\section{Volumen 24 № 1 Enero-Abril 2020}

$(222-244)$

LA MEDIACIÓN COMO ESTRATEGIA DE RESOLUCIÓN DE CONFLICTOS PACÍFICA EN EL ÁMBITO ESCOLAR

MEDIATION AS A PACIFIC CONFLICT RESOLUTION STRATEGY IN THE SCHOOL SCOPE

\section{Resume}

El objetivo del presente estudio fue analizar la mediación como estrategia de resolución de conflictos pacifica en el ámbito escolar. Metodológicamente el desarrollo de este trabajo, se llevó a cabo mediante una investigación de tipo documental y diseño bibliográfico, la técnica consistió en el análisis documental o contenido. Las fuentes ubicadas fueron de carácter documentales, tales como bibliográficas, hemerográficas o archivísticas; la primera se basó en estudios sobre la teoría de conflicto. En conclusión, la incorporación de la mediación escolar como estrategia de resolución de conflictos en el ámbito escolar, necesitará de un compromiso, así como nivel de implicación importante a todos los miembros de la comunidad educativa; comenzando por el equipo directivo, profesores, alumnado, los padres y el personal no docente.

Palabras Clave: mediación; estrategia; resolución de conflictos; ámbito escolar.

\begin{abstract}
The objective of this study was to analyze mediation as a strategy for the resolution of peaceful conflicts in the school environment. Methodologically, the development of this work was carried out through a documentary research and bibliographic design, the technique consisted of the documentary analysis or content. The sources located were documentary, such as bibliographic, hemerographic or archival; The first was based on studies on conflict theory. In conclusion, the incorporation of school mediation as a conflict resolution strategy in the school environment will require a commitment, as well as an important level of involvement to all members of the educational community; starting with the management team, teachers, students, parents and non-teaching staff.
\end{abstract}

Keywords: mediation; strategy; conflict resolution; school environment. 


\section{INTRODUCCIÓN}

Entender que el ser humano es un ser social por naturaleza, implica reconocer que su desarrollo se da en la constante interacción con los otros. En las relaciones humanas cada individuo tiene unos intereses, aptitudes, actitudes personales, las cuales influyen y puede llevar a que se presente una convivencia positiva o negativa. La convivencia para Mockus, (2002) es el acto en el que se deben "acatar reglas comunes, contar con mecanismos culturalmente arraigados de autorregulación social, respetar las diferencias y acatar reglas para procesarlas, de la misma manera es aprender a celebrar, a cumplir y reparar acuerdos" (p. 2).

La escuela cumple la función de formar sujetos sociales, fortaleciendo en los estudiantes competencias que les permitan desenvolverse adecuadamente en sociedad, respetando las diferencias, aceptando y dando cumplimiento a las normas establecidas. Sin embargo, en ocasiones se presentan situaciones que amenazan la convivencia en las instituciones educativas, los procesos de mediación como sistemas de resolución de conflictos pueden verse como la consecuencia lógica a una realidad compleja para obtener los objetivos deseados, las relaciones interpersonales como base de una convivencia pacífica, no violenta y respetuosa con los demás.

La mediación es una técnica de resolución de conflictos que puede ser muy útil para combatir los nuevos retos que se presentan en la sociedad actual, caracterizada por el cambio constante. Viana (2015) la define como "una herramienta al servicio de la convivencia pacífica que abre paso a una nueva cultura basada en el diálogo como estrategia de transformación de los conflictos" (p. 41).Su potencial para generar aprendizaje en cualquier persona por el hecho de participar en un proceso de mediación es evidente, su extensión a todos los ámbitos de interrelación humana (familiar, penal, comunitario, intercultural, empresarial, político, entre otros.).

En consecuencia, se debe ser consciente de que uno de los cometidos del sistema educativo es precisamente la socialización de los individuos, pues las desavenencias constituyen un importante punto en las relaciones sociales; en este sentido se cree que un aspecto fundamental de la educación es enseñar a los alumnos a resolver sus diferencias de manera adecuada, sobre todo teniendo en cuenta que en la actualidad se tilda a la juventud de violenta.

La misma, se apuesta según Ridao (2008) "por una perspectiva antropológica de la educación, es decir, enseñar desde el razonamiento de que todos somos más iguales que 
diferentes" (p. 40); o sea, en ningún momento se trata de acentuar las discrepancias que caracterizan a cada una de las culturas. En los centros educativos se dan conflictos de muy distinta índole: relaciones con los alumnos, superiores de trabajo e incluso entre las instituciones familia, escuela.

Como consecuencia, se puede distinguir entre las mediaciones simétricas y las asimétricas, muy especialmente en los colegios, dándose mediaciones entre pares, cuando los niños son los propios mediadores, las mediaciones utilizadas para resolver conflictos dentro de la escuela, donde los mediadores pueden ser profesionales o personal administrativo del colegio instruidos en esta técnica.

Dicho de otro modo, la mediación es un procedimiento, compuesto por una serie de estrategias y técnicas, mediante el cual las partes implicadas, con la participación ayudan al mediador imparcial e independiente a identificar, formular, analizan las cuestiones en disputa, con el objetivo de desarrollar, proponer ellas mismas opciones o alternativas que les permitan llegar a un acuerdo que solucione el conflicto o mejore las relaciones entre las partes.

En tal sentido, una premisa básica de la mediación es la voluntariedad de las partes en acudir al mediador para embarcarse en el proceso, así como la aceptación sin reservas del que media como persona imparcial e independiente sin ninguna relación con el problema o conflicto que las enfrenta, de manera que lo perciban como una figura que les va ayudar a encontrar un acuerdo, pero nunca como un enemigo ni tampoco como un aliado. Raposo (2012), la define como:

Voluntariedad, tanto para el inicio, como para el desarrollo y finalización, si bien la falta de asistencia a una primera sesión informativa indicada por el Tribunal puede considerarse como mala fe procesal, debiendo el mediador informar al Tribunal que parte o partes no acudieron. (p. 1)

De tal manera, junto a las mediaciones educativas en contextos interculturales, como ya se ha visto, se debe tener en cuenta que también se ha desarrollado la mediación en centros escolares donde todos los alumnos comparten las mismas costumbres culturales, puesto que se han realizado diversas investigaciones en escuelas de primaria con el fin de averiguar las técnicas que utilizan los niños para resolver sus conflictos antes de conocer la mediación; dichos análisis han demostrado que los estudiantes empleaban la fuerza física, la agresión y la retirada, o se lo contaban al profesor. 
Al respecto, Viñas (2004) advierte de que "con frecuencia los conflictos graves tienen un largo pasado, por lo que hay que zanjar tales discordias por completo en el menor tiempo posible, aparte de que también se debería trabajar la prevención de tales episodios de desavenencia"(p. 14). Esta situación la lleva al contexto específico de la educación, pues señala que algunas situaciones conflictivas que se encuentran en los últimos cursos de escolaridad obligatoria han ido gestándose durante años, al tiempo que una buena parte de estos problemas surgen como consecuencia de los procesos de autoafirmación durante su desarrollo como persona.

En relación con el presente estudio, el iniciar el camino de reflexión para analizar la mediación como estrategia de resolución de conflictos pacifica en el ámbito escolar, implicó una revisión de diversas fuentes de información, así como posturas teóricas de diversos autores, que permitieron el proceso indicativo. La intención no fue presentarlo como un basamento teórico que estableció la racionalidad del problema, lo que requirió dejar en claro, su construcción a través del conocimiento que emergió a lo largo de la investigación.

\section{TEORÍA DE CONFLICTO}

El conflicto es un fenómeno natural en toda sociedad, es decir, se trata de un hecho social consustancial a la vida en sociedad. Así mismo, las disputas son una constante histórica, puesto que han comparecido en todas las épocas en sociedades a lo largo de los tiempos. Incluso, el cambio social que determina toda la dinámica de la vida de los seres humanos es una consecuencia que debe ser imputada de modo mayoritario, aun cuando no de manera absoluta, a la disputa. Aparece como una parte natural de las relaciones interpersonales. Es difícil concebir las interacciones de las personas sin que se presente un conflicto. Pardo (2014) considera al respecto que "el conflicto es parte inevitable del proceso de crecimiento y desarrollo de cualquier grupo social y del ser humano" (p. 1).

Además, (Castells, citado por Rodríguez (2007) señala "el conflicto forma parte de la vida y afecta a todos los ámbitos de nuestra existencia” (p. 1). Siempre que haya divergencias en los intereses, opiniones y necesidades o cualquier tipo de incompatibilidad, se generará una oportunidad para un desacuerdo o disputa. Es importante entonces recurrir a una variedad de acciones y métodos que permitan canalizar cada situación conflictiva de forma que desemboquen en pactos favorables para los actores involucrados. 
Así mismo, la solución no debe ser impuesta por terceras personas. El conflicto se entiende como el rompimiento de la comunicación por discrepancia entre dos o más personas, lo que impide llegar a acuerdos o al consenso. Estas discrepancias se convierten en el ambiente escolar, en disputas que se manifiestan a través de comportamientos: gestos, apodos, palabras, rechazo y en ciertos casos en agresiones más fuertes como los golpes. El sentido humano, al que hace referencia Galtung (2003), que es una especie de categoría más elevada como un patrimonio universal, invita a salir, mirar más allá de dualismos y etnocentrismos. Así, desde un diálogo con imaginarios, así como filosofías orientales concluye que es una constante en la historia humana la trilogía: paz-violencia-humanidad.

En tal sentido, importa resaltar dos ideas base que están detrás de esta formulación y que van dando las primeras pistas de la perspectiva antropológica de Galtung (ob. cit): "Primero que la complejidad humana, requiere respuestas igualmente complejas" (p. 63). Para ello es oportuno romper con esquemas tanto dualistas como simplificadores para abrirse a otro tipo de racionalidad que puede iniciar por ejemplo con los conceptos triádicos, típicos del autor. Las concepciones del hombre son un criterio hermenéutico práxico de la historia y las civilizaciones. En ese entender no hay una sola noción, sino varias.

Seguidamente, una segunda afirmación, siempre en relación al fundamento antropológico de la paz, es que el "hombre es un ser con capacidad de paz” (p. 64). Para llegar a tal afirmación el autor se pone en debate con cuatro teorías de la evolución, que, a su juicio, son las más representativas. El hace una crítica a cada una de estas, pero luego plantea desde una síntesissuma de todas estas, su propia teoría. La cuestión aquí es si fue el hecho de la cooperación o competencia lo que lleva al hombre a acercarse y cumplir su destino final, a evolucionar.

Al respecto, está claro el principio de que una teoría de conflictos, no sólo debe reconocer si estos son buenos o malos; esta deberá fundamentalmente ofrecer mecanismos para entenderlos lógicamente, criterios científicos para analizarlos, así como metodologías (creatividad, empatía y no violencia) para enfrentarlos. El bienestar se concibe más como suelo que como techo, porque cuanto más se detalla, más rica y específica es su definición. De allí la importancia de conocer, comprender la naturaleza del conflicto que se presenta en el ámbito educativo para aplicar las estrategias adecuadas que permitan resolverlo de manera pacífica y positiva.

Cabe destacar, en Galtung (ob. cit), es de suma importancia contar con una teoría del conflicto tanto para los estudios para la paz en su versión negativa, como reducción de la 
violencia directa, del sufrimiento u ofensa a las necesidades humanas básicas. Sobre el desarrollo, se proyecta ir más allá de la satisfacción de las necesidades humanas básicas, buscando propuestas, estrategias para desarrollar, potenciar esas mismas necesidades. Se apoya, en una perspectiva antropológica en las que las concepciones del hombre son un criterio hermenéutico de la historia de las civilizaciones, entendiendo que no hay una sola historia sino historias, donde los conflictos se resuelven por medios pacíficos pues el hombre es un ser con capacidad de paz.

En otras palabras, los aportes de esta teoría, son las lecciones que el referido autor afirma reiteradamente haber aprendido en sus ya 50 años de trabajo como investigador sobre la paz (Oslo, enero de 1959) al señalar que las teorías de los conflictos son para la eternidad. Evidentemente esta constatación bastante realista, en medio de un mundo en constante cambio, tiene que empujar a una redefinición constante de las categorías mentales y por ende de las concepciones científicas que son los medios con los cuales se acerca, conoce, comprende la realidad en la que se vive.

\section{La mediación como estrategia de resolución de conflictos}

La mediación es un proceso de resolución de conflictos en el que las dos partes enfrentadas recurren voluntariamente a una tercera persona imparcial, el mediador, para llegar a un acuerdo satisfactorio. Para Rozenblum, (1998) "es un proceso extrajudicial o diferente a los canales legales o convencionales de resolución de disputas" (p. 126), es creativo, porque mueve a la búsqueda de soluciones que satisfagan las necesidades de las partes, e implica no restringirse a lo que dice la ley. Además, la solución no es impuesta por terceras personas, como en el caso de los jueces o árbitros, sino que es creada por las partes.

Cabe considerar, como una de sus características a la negociación cooperativa, en la medida que promueve una solución de las partes implicadas gana u obtienen un beneficio, y no sólo una de ellas. Por eso se la considera una vía no adversarial, porque evita la postura antagónica de ganador-perdedor. Por este motivo, según Holaday, (2002) "también es un proceso ideal para el tipo de conflicto en el que las partes enfrentadas deban o deseen continuar la relación" (p. 126).

En tal sentido, para que el proceso de mediación sea posible, es necesario que las partes estén motivadas, porque deben de estar de acuerdo en cooperar con el mediador para resolver su disputa, así como para respetarse mutuamente durante y después del proceso, así como 
respetar los pactos que se hayan alcanzado, circunstancia que ocurre con un alto índice de cumplimiento, porque son los que los mismos interesados han propuesto comprometidos.

En este orden de ideas, la mediación en conflictos para Likert y Likert (1976) y Shapiro (2002)se constituye como un proceso ordenado que se inicia por la pérdida de la capacidad negociadora de las personas en conflicto. Las partes están encerradas en sus posiciones y gracias a la ayuda un tercero, que no se implica en la solución, se genera un nuevo espacio de comunicación, a la vez de aprendizaje, para ambas partes. Esta característica de aprendizaje, por lo tanto, de transformación de la persona, es una de las claves para incorporar esta estrategia al espacio educativo.

Por tanto, la mediación como estrategia de resolución de conflictos se puede situar entre el compromiso y la colaboración. La finalidad consiste en pasar de estilos más individualistas a modos más evolucionados. También puede suceder que, para crear un contexto real de cooperación, sea necesario pasar por momentos previos en los que se manejen estilos como el de evitación o acomodación, ya que pueden ser necesarios para generar un nivel de confianza entre las partes, imprescindible para llegar a la ayuda esperada. De tal manera, para Rozenblum (ob. cit):

Los procesos de mediación como sistemas de resolución de conflictos pueden verse como la consecuencia lógica de una realidad compleja en la que las relaciones de convivencia a nivel social, familiar, laboral, escolar, entre otras, han sufrido variaciones muy profundas, así como rápidas en las últimas décadas. Las formas clásicas de regulación de la tolerancia no parecen ser suficientes ante la diversidad de visiones en una situación de conflicto. (p.128)

En efecto, parece lógico, que, desde el ámbito educativo, los profesionales de la educación intenten dotar a sus alumnos, de formas de gestión de conflictos del siglo XXI. Adaptadas a las nuevas dinámicas de coexistencia actuales que exigen, no sólo obtener los objetivos deseados, sino también mantener las relaciones interpersonales como base de una convivencia pacífica, no violenta y respetuosa con los demás. La convivencia en la escuela puede considerarse como un reflejo de cohabitación en la sociedad.

Al respecto, la complejidad de las relaciones interpersonales actuales genera conflictos de cuya gestión, así como resolución, dependerá la existencia de adecuados climas de convivencia en los centros educativos. Prada y López (2008) exponen "la utilización de la mediación escolar como sistema de resolución de conflictos es la consecuencia lógica ante la nueva realidad, cambiante y plural, de las escuelas" (p. 101). El enorme potencial que presenta esta 
estrategia de resolución pacífica de conflictos a nivel escolar queda perfectamente expresado en la siguiente frase de Munné y Mac-Cragh (2006.)

La mediación abre los ojos a una realidad compleja; a entender que las cosas no son nítidas ni iguales para todos; a pensar que se pueden cambiar las percepciones sin dejar de ser uno mismo y rehacer las expectativas iniciales e individuales por expectativas conjuntas; a ver que para la comprensión ajena se necesita la autocomprensión, el autoconocimiento; a saber que elconflicto es propio de todos, por tanto, tiene aspectos tanto positivos como negativos; a creer que las cosas pueden mejorar con la colaboración y a confiar en que ésta tenga un potencial de cambio social. (p. 6)

Incluso, la mediación escolar no sólo es una estrategia, sino que conlleva una serie de valores, procedimientos que educan en la cultura de la paz, consolida formas de actuación, gestiones de las disputas profundamente participativas y democráticas. Estos elementos de carácter actitudinal le confieren unas peculiaridades muy interesantes para ser una excelente locomotora que haga moverse todo un tren de iniciativas para la mejora de la convivencia en un centro educativo, es decir, puede dar la fuerza y el poder de consolidación necesarios a todo un proyecto de entendimiento escolar en la escuela.

Por consiguiente, este tipo de proyecto o plan, se compondrá de muchos procesos, actuaciones en diferentes aspectos de la vida de centro, pero deberá poseer algún elemento identificador que haga que todo el plan camine hacia adelante. La creación de equipos de mediación escolar, formados por miembros de todos los sectores de la comunidad educativa, puede ser el elemento dinamizador de la convivencia escolar. Uno de los modelos que más se adapta al ámbito escolar, desde la perspectiva del trabajo de la convivencia, es el transformativo. Siguiendo a Léderach (2002), las fases de este proceso de mediación serían las siguientes:

a)Premediación: En esta fase se realiza una reunión por separado con cada persona en conflicto, en busca de una descarga emocional previa a la mediación conjunta. Se trata de la primera toma de contacto y conocimiento de los mediadores as y cada parte. Se explica el mecanismo de la mediación y se hace un relato del conflicto y sus consecuencias.

b)Entrada: Se realizan las presentaciones y se explican las condiciones y normas para poder realizar la mediación.

c) Cuéntame: Cada una de las partes en conflicto relata lo que ha sucedido.

d)Situar el Conflicto: Se realiza un análisis del conflicto, resaltando los aspectos en común que han expuesto las partes. Se pueden pedir aclaraciones. 
e)Buscar Soluciones: Se intenta realizar un nuevo enfoque para avanzar hacia la solución. Se pide a las personas en conflicto, por parte de los mediadores-as, que realicen un torbellino de ideas sobre posibles soluciones al conflicto.

f)El Acuerdo: Se elige una solución, se analiza, y se redacta un acuerdo para su firma.

En fin, es evidente que si las personas en conflicto no están formadas en determinadas habilidades comunicativas (escucha activa, mensajes yo, asertividad, entre otras), será labor de los mediadores conseguir que durante el proceso vayan utilizándolas, en la medida de lo posible. En este sentido, adquiere una enorme importancia la fase previa de premediación, donde las personas en pugna tienen un primer acercamiento a estas actitudes de comunicación relación, que constituyen la base del proceso mediador y de la mediación como estrategia formativa, de transformación de las personas.

\section{Importancia de la mediación como estrategia de resolución de conflictos}

Previamente al análisis de la importancia de la mediación como estrategia de resolución de conflictos pacifica en el ámbito escolar es necesario recordar algunos principios básicos en relación con el conflicto y la mediación. Los mismos, deben ser situados dentro del contexto más amplio de las relaciones interpersonales, puesto que la educación es según Fernández (2004) “en esencia, un fenómeno interpersonal en el que la calidad de la relación entre educador y educando, y entre el propio alumnado, resulta clave para el propio proceso de aprendizaje" (p. 4).

De hecho, diversos programas centrados en el desarrollo de competencias sociales en alumnos y profesores han demostrado ser un medio eficaz para mejorar no sólo la convivencia sino también el rendimiento académico, así como la propia autoestima de los alumnos. Sin embargo, en este ámbito, como en otros también caracterizados por la continua interacción entre quienes en ellos se desarrollan, éstos no pueden ser totalmente eliminados, ni probablemente sea conveniente que lo fueran.

En este sentido, conviene recordar que los conflictos, cuando se afrontan adecuadamente, son un elemento importante de evolución, cambio para las personas, así como para los grupos. Delimitando con algo más de precisión el término conflicto, cabe señalar que éste surge cuando dos actividades resultan incompatibles a una o varias personas, es decir, cuando la realización de una de interés, necesidad o motivación interfiere con otra en la misma persona, o de otra u otro sujeto. 
Considerando lo antes plantado, pueden ser de tipo intrapersonal (cuando una misma persona percibe deseos o necesidades incompatibles), interpersonal (dos personas con intereses, deseos o necesidades contrapuestos e incompatibles), intergrupal (la incompatibilidad percibida surge dentro de un grupo, organización, institución o nación). No obstante, dentro del ámbito escolar las pugnas de tipo interpersonal es el que ha sido objeto de mayor análisis e investigación debido a su mayor relación con los problemas de disciplina escolar. Una de las definiciones más conocida es la de Torrego (2000), quien lo define como:

Aquella situación en la que dos o más personas entran en oposición o desacuerdo porque sus posiciones, intereses, necesidades, deseos o valores son incompatibles, o son percibidos como incompatibles, donde juegan un papel muy importante las emociones, sentimientos y donde la relación entre las partes en conflicto puede fortalecerse o deteriorarse en función de cómo sea el proceso de resolución del conflicto. (p. 37)

Ciertamente, los conflictos constituyen un elemento esencial e inevitable de las relaciones interpersonales que incluyen aspectos negativos (un posible desgaste, el deterioro de la relación, escaladas en el enfrentamiento o la imposibilidad de llegar a una solución), pero también aspectos positivos (posibilitan el cambio, el crecimiento y la madurez). El hecho de que las diferentes formas de afrontar una diputa interpersonal tengan consecuencias tan diferentes, unido a que éstos son inevitables, justifica plenamente que se preste una mayor atención desde los centros escolares tanto a las estrategias de manejo adecuado del conflicto como a la posible creación de equipos de mediación.

De modo que, la prevención de disputas en el ámbito escolar no surge por generación espontánea. Requiere de una decisión pedagógica. Se deben pensar las condiciones acerca de las técnicas alternativas de solución de conflictos y desarrollar estrategias de intervención en la escuela. Una gestión del centro educativo, que tenga como norte la mejor convivencia dentro del ámbito escolar, sin duda impactará positivamente el clima social de éste. Aprender a vivir juntos debe formar parte de lo que se entiende por calidad educativa. Aprender a vivir juntos es una necesidad, más que un problema.

Por eso, de la decisión del equipo directivo del centro educativo depende la creación de grupos de mediación entre pares, también la preparación docente para detectar en forma temprana los casos de maltrato y violencia en la comunidad educativa y los esquemas de negociación para que las partes involucradas resuelvan sus tensiones. Entre las principales técnicas de resolución de conflictos Moreno y Sastre (2010) cita las siguientes: 
(a) Mediación: Procedimiento en el cual dos partes de un conflicto se reúnen con un tercero, ajeno e imparcial, que facilita la comunicación entre aquellas para que puedan delimitar el conflicto y encontrar su solución. El tercero no hace propuestas de arreglo. (b) Conciliación: Procedimiento en el cual dos partes de un conflicto se reúnen con un tercero, ajeno e imparcial, que facilita la comunicación entre las personas enfrentadas para delimitar y solucionar el conflicto, y que además formula propuestas de solución. (c) Arbitraje: Procedimiento en el cual un tercero, ajeno e imparcial que no cumple funciones de juez público y que ha sido nombrado o aceptado por las partes, resuelve un litigio mediante una decisión vinculativa y obligatoria (p. 26).

Como resultado, estos cuatro medios alternativos presentan como común denominador la no intervención de un juez público, o al menos, su intervención no con facultades decisorias en el caso de la mediación y la conciliación intraprocesales. Para efectos de esta investigación, se hará referencia sólo a la mediación como estrategia de resolución de conflictos pacifica en el ámbito escolar

\section{Estrategias básicas para la mejora de la convivencia y la resolución de conflictos en el ámbito escolar}

La escuela permite el aprendizaje, la práctica de valores democráticos como la solidaridad, justicia y paz, traducidos en la cotidianeidad transcurrida en el aula. Derivada de la condición natural de ser social, la socialización innata del hombre acarrea el concepto de convivencia, el que resulta cotidiano a las personas, teniendo su primera aproximación en el seno de la familia. Ortega y Martín (2004) exponen "La Real Academia de la Lengua Española, hace referencia al término convivencia como la acción de vivir juntos, acción que se puede contextualizar desde los contextos del habla popular, el contexto psicológico y el contexto jurídico social” (p. 19)

En el contexto del habla popular la definición del término convivencia dice relación con aquellos que comparten un lugar físico, también un sistema de normas que ordenan la vida conjunta para evitar el surgimiento de conflictos y si llegan a producirse éstos, la relación de convivencia permite su resolución equitativa justa. Diversas investigaciones Cascón (s.f.), Chaux (2002), Viñas (2004) entre otras, plantean la cultura y resolución pacífica de conflictos como estrategias para mejorar la convivencia escolar.

De ahí que, la escuela debe favorecer, proveer habilidades, conocimientos, competencias que les permita a los estudiantes enfrentar situaciones de disputa o divergencia. Los estudios coinciden en que para mejorar la convivencia escolar es necesario un proceso de formación ciudadana, la comunicación y en particular el diálogo, al igual que el manejo de las emociones, 
pensar antes de actuar para no dejarse dominar por la rabia son premisas fundamentales a la hora de enfrentar un conflicto.

En este entorno, la mediación se ha convertido en una alternativa para prevenir, así como resolver conflictos escolares, que permite repensar las relaciones interpersonales, reconocer las consecuencias de los propios actos, manejar el enojo y la rabia personal para entablar con el otro una relación cordial. Prada y López (ob. cit) proponen las siguientes características de la mediación escolar: es voluntaria (por decisión), es estrategia pacífica, es sistemática a través de fases de desarrollo, favorece el proceso de comunicación y colaboración humana, facilita el encuentro a través de los mediadores, es transformadora de las personas, promueve la formación de quienes participan, las personas aprenden a gestionar sus conflictos y es confidencial. En efecto, estas características resaltan las bondades de la mediación, sus alcances, así como las personas involucrados. En este mismo sentido, Viñas (ob. cit) propone que:

en la implementación de la cultura sobre la temática, se debe construir relaciones, fomentar la comunicación, trabajar las tres R de Galtung (ob. cit): Reconciliación, Reconstrucción, Resolución, adecuar la dinámica, progresión del conflicto, crear aulas pacíficas para adquirir pautas elementales de convivencia, definir los planteamientos institucionales en relación a la convivencia, reestructurar la cultura, las políticas, las prácticas de las instituciones educativas para que puedan atender a la diversidad y multiculturalidad (p. 65).

De igual manera Cascón (s.f.) propone abordar los conflictos en su primera fase, para buscar soluciones sin apasionamientos, poderlos analizar y no enfrentarlos de una forma violenta, se debe buscar la solución no violenta. En la escuela, se deben aprovechar distintos momentos como las clases, descanso, horas específicas de formación en competencias ciudadanas, dentro de un programa específico o ante la necesidad de conflictos reiterados. El citado autor, propone que "trabajar el conflicto en tres niveles consecutivos: prevención, negociación, mediación (...) la prevención a nivel educativo va a significar intervenir en el conflicto cuando está en sus primeros estadios, sin esperar a que llegue la fase de crisis" (p.14).

De manera pues, en la mediación se recurre a una tercera parte que ayudará en el proceso transversal a estos niveles está la acción "no violenta", se trata solucionar el conflicto asertivamente, defendiendo los derechos y respetando los derechos del otro. Se ha planteado la necesidad de desarrollar, potenciar habilidades, así como estrategias que permitan a los 
estudiantes enfrentar mejor los conflictos. Cascón (s.f.) propone un proceso que cree las bases para enfrentar dichas situaciones (Figura 1).

Figura 1

Proceso para formar en la resolución de conflictos

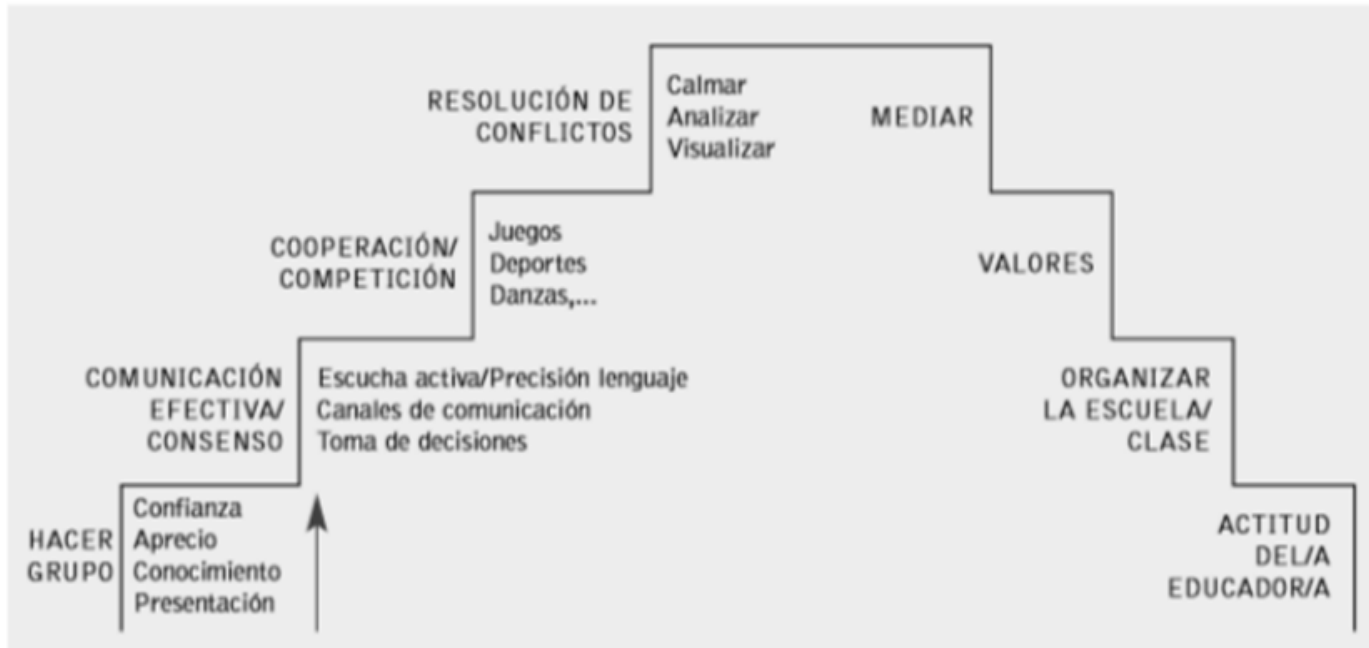

Nota: Tomado de Cascón (s.f.).

En la figura 1 se observan dos escaleras, en la del lado izquierdo, están planteados los temas a trabajar con los estudiantes, en la derecha las implicaciones que tiene para los docentes. La educación debe fortalecer, formar valores de convivencia, solidaridad, diálogo, que permitan desarrollar acciones encaminadas a la generación, desarrollo de habilidades para la paz y la no violencia. Los conflictos para Chaux (ob. cit) "deben ser utilizados como oportunidades para incentivar el diálogo, dar cambios a las relaciones, fortalecer la capacidad de ponerse en el zapato del otro (p. 36).

Por consiguiente, para la solución del conflicto es necesario que las partes tengan conciencia de la existencia del mismo, buscar acuerdos que conlleven a la resolución. Por esto, es necesario que aprendan a manejarlos porque son parte de la vida y en algunos casos son inevitables. Según la teoría existen cuatro formas de manejar las pugnas: evitar las personas con quien se tiene el conflicto; imponer las ideas propias, sin tener en cuenta la persona con quien se tiene la pugna; renunciar a las ideas propias, para no afectar la relación; buscar acuerdos que favorezcan los intereses propios sin afectar la relación con el otro. 
En este sentido, para Chaux (ob, cit) en sus investigaciones "evidencia que los estudiantes que han recibido formación en resolución de conflictos, adoptan las dos primeras formas para enfrentar el conflicto" (p. 37); esto demuestra la necesidad de formar al colectivo educativo en esta temática, para que a través del proceso desarrollen habilidades con el fin de mejorar el clima escolar, formando a toda la comunidad educativa en el manejo de enfrentamientos en búsqueda de una convivencia pacífica para todos.

\section{La comunicación efectiva como estrategia de mediación para la resolución de conflicto en el ámbito escolar}

La función del docente en el proceso de enseñanza-aprendizaje, tiene como objetivo buscar las estrategias necesarias para desarrollar mejor su actividad, entre las cuales debe tomar en cuenta las características que manifiestan y observan los alumnos. Por ello, debe tener dominio del tema, el uso adecuado de las estrategias pedagógicas, ser un docente interactivo, creativo, motivador, facilitador del aprendizaje integral, abierto a la comprensión de los demás, a la cooperación, respeto mutuo e indagación permanente. Esto lo puede lograr a través de la comunicación, de la cual se van a desprender las técnicas de escuchar, legitimación, parafrasear, resumir y motivar.

Cabe destacar que, la comunicación como un proceso, ha sido estudiada por muchos investigadores quienes han determinado que el origen de la misma es latino, deriva del adjetivo communin, que significa algo que es poseído solidariamente por varias personas. Al respecto, Griffin (2011) "la comunicación efectiva es el proceso de enviar un mensaje en forma tal que el mensaje recibido sea tan cercano en significado como sea posible al mensaje emitido" (p. 586).

De tal manera, la función esencial de un sistema de comunicación es la de crear una comprensión integral del trabajo cooperativo no sólo en relación con los fines sino también con las normas, hábitos, creencia en la escuela. Para que esto sea factible, debe haber una transmisión de significados entre sus miembros. Sólo mediante la transmisión de significado de una persona a otra se puede compartir la información, ideas, valores, costumbres, hábitos, entre otros. Ahora bien, para que la comunicación dentro de la escuela sea efectiva, Fuenmayor (2012), debe cumplir con ciertos requisitos: claridad o lenguaje accesible, integración para los miembros de la comunidad, aprovechamiento de la organización informal, equilibrio entre las acciones y la comunicación del plan, moderación de la comunicación (concisa y necesaria), difusión por escrito, evaluación periódica de los sistemas y canales de comunicación. 
Por otra parte, se debe tener en cuenta el factor fundamental con respecto a la comunicación efectiva en el ámbito escolar, es el hecho de que las escuelas están compuestas por personas, las personas se relacionan entre ellas precisamente, a través del lenguaje. Al respecto, señala Cardoso (2014) que "las relaciones, así como las comunicaciones humanas son indivisibles porque es imposible llevar a cabo unas sin las otras" (p. 36). Cuando dos o más personas coinciden en este lugar, automáticamente se puede iniciar un mensaje, lo que conlleva a una reacción emocional, posiblemente, a una interrelación personal.

Cada uno puede expresar una opinión, un sentimiento acerca de algo, acerca del otro, o de otros. Entonces, se crea un lazo. Una vez que se habla con alguien, se crea un vínculo, algo se da, recibe, cambia. Es el poder que tienen las palabras para bien y para mal. Dentro o fuera del contexto escolar, los seres humanos son sensibles a las palabras, a lo que otros dicen. Y esto es algo que un mediador debe saber, para evitar conflictos o para solucionarlos, usar las palabras en sentido positivo, o asertivo.

De acuerdo a las definiciones anteriores, la comunicación es una relación entre dos o más personas, de participación mutua, de encuentro, donación, referencia al ser- si -mismo, el transmitir mensajes, sentimientos y pensamientos, todo esto con lleva a la manera cómo se interprete, esto está vinculado con sus propias percepciones, creencias y experiencias personales. Por consiguiente, para que una comunicación sea realmente efectiva, debe crear una atmósfera sana, que estimule el respeto, la confianza, la motivación, el logro de las metas y la aceptación recíproca.

Al respecto, Habermas citado por Arráez (2010), afirma que la validez de la acción comunicativa depende del intercambio permanente de conocimientos y experiencias para clarificar el poder de decisión, el compromiso de la comunidad o institución en la gestión, programación, desarrollo de acciones conjuntas, fundamentadas en la comunicación, delegación de las funciones para la identificación, solución de problemas comunes en la transformación de realidades educativas, específicas así como concretas. Por lo cual, se hace primordial destacar la necesidad de escuchar para poder ser flexible e ir cambiando y ampliando las percepciones.

Es por ello que, saber escuchar es un ingrediente esencial al momento de abordar y resolver conflictos; escuchar permite obtener información útil de la persona que nos está hablando. Según Cuenca (2009) señala, que el escuchar es un proceso que implica el estar aquí y ahora, 
presente en el instante por lo que se hace difícil que se esté pensando en otra cosa e inclusive el pasar el tiempo en pensar lo que se va a contestar o responder al interlocutor, al cual, de igual manera, se le debe responder en el mensaje de los sentimientos; el tener paciencia, mirarle de frente y con toda la atención a la persona con la que se está hablando.

De allí, que el escuchar de manera activa consiste en la disposición plena de focalizar toda la atención en la otra parte, captando con todos los sentidos lo que expresa, cómo lo expresa (comunicación verbal, para-verbal, no verbal, contextual), cuáles son sus necesidades subyacen a las posiciones. Para Maturana (2002) "al escuchar a las personas se les ofrece un espacio de aceptación y respeto que da sentido y legitimidad social a sus vidas” (p.110). Otra técnica aplicable en la comunicación es la legitimación la cual señala que es la disposición a encontrar internamente y reconocer externamente cualidades positivas de cada parte frente a sí misma empoderamiento y entre ellas reconocimiento. Para Cuenca (ob. cit), la legitimación es encontrar razones positivas para hacer sentir bien a las partes, en forma genuina y honesta, a través de una frase o comentario en el momento oportuno.

También, está el parafrasear como otra técnica, definida como una síntesis verbal que refleja en un lenguaje neutro lo dicho por las partes, en este contexto el autor antes citado establece que el lenguaje neutro protege a las partes de las inclemencias verbales, para verbales, así como no verbales de las discusiones, de las intenciones de ofender o hacer daño al calor de las emociones.

Así mismo, el resumir como técnica de la comunicación, es una síntesis verbal que refleja lo dicho por las partes, en cuanto a ideas centrales en el transcurso del diálogo expresada mediante reformulaciones y connotaciones positivas. Establece el mismo autor; el resumir contribuye a ordenar ideas, centrar los asuntos fundamentales y proporciona un cierre cognitivo a la sesión de mediación.

Igualmente, se menciona la motivación a modo de técnica de comunicación la cual es el motor que impulsa a la acción, para la satisfacción de las necesidades. McClelland (1965) y Atkinson (1964) señalan, "El motivo es una asociación afectiva y se manifiesta como conducta intencional. Toda motivación se basa en emociones" (p. 136). Se infiere que las tendencias emocionales guían o facilitan la obtención de las metas. Finalmente, cada uno de estos aspectos abordados son algunas de las estrategias de mediación, las cuales brindará al docente 
herramientas pertinentes y eficaces para influir, transformar, sensibilizar y construir un sistema cognitivo emocional adaptado a nuevas realidades.

Cuando en las intervenciones de mediación se fomenta un clima que lo favorece, para Torrego (2003) las partes en disputas se sienten atendidas participan en el diálogo. La escucha activa empática se basa en el resumen por parte del mediador de las expresiones, posturas de cada una de las partes, recogiendo en este resumen el estado anímico de implicación en el conflicto. Al recopilar, no sólo los hechos, sino los sentimientos de las partes el mediador muestra que entiende y comprende la explicación y postura de cada una de ellas; conocido también como parafrasear.

En tal sentido, se puede decir que se practica la escucha activa cuando el receptor entiende lo dicho y destaca el sentimiento que hay detrás del mensaje, es decir, se manifiesta de forma no verbal (por ejemplo, moviendo la cabeza en sentido afirmativo), o de forma verbal mediante la emisión de palabras neutras que no muestren aprobación ni desaprobación (por ejemplo, ¿puedes explicarme más esto?). A continuación, se presentan el Cuadro Técnicas para la Escucha Activa.

\section{Cuadro 1}

Técnicas para la Escucha Activa

\begin{tabular}{|c|c|c|c|}
\hline Técnicas & Objetivos & Procedimientos & Ejemplo \\
\hline Mostrar interés & $\begin{array}{l}\text { Comunicar interés } \\
\text { Favorecer que el otro } \\
\text { hable }\end{array}$ & $\begin{array}{l}\text { No estar en acuerdo ni } \\
\text { en desacuerdo } \\
\text { "Utilizar palabras } \\
\text { neutrales" }\end{array}$ & $\begin{array}{l}\text { "¿Puedes contarme } \\
\text { más sobre esto?" }\end{array}$ \\
\hline Clarificar & $\begin{array}{l}\text { Aclarar lo dicho. } \\
\text { Obtener } \\
\text { más } \\
\text { ver losmación Ayudar a } \\
\text { vista }\end{array}$ & $\begin{array}{l}\text { Preguntar Pedir que } \\
\text { aclaren algo que no } \\
\text { has entendido }\end{array}$ & $\begin{array}{l}\text { "¿Y tú qué hiciste en } \\
\text { ese momento?" } \\
\text { "¿Desde cuándo estáis } \\
\text { peleados?" }\end{array}$ \\
\hline Parafrasear & $\begin{array}{l}\text { Demostrar que } \\
\text { estamos } \\
\text { comprendiendo to que } \\
\text { pasa Verificar el } \\
\text { significado }\end{array}$ & $\begin{array}{l}\text { Repetir las ideas y } \\
\text { hechos básicos }\end{array}$ & $\begin{array}{l}\text { "Entonces, para ti el } \\
\text { problema es una } \\
\text { tonteria" "Entonces, } 10 \\
\text { que me estás diciendo } \\
\text { es..." }\end{array}$ \\
\hline Renlejar & $\begin{array}{l}\text { Mostrar que se } \\
\text { entienden los } \\
\text { sentimientos Ayudar a } \\
\text { que la otra persona sea } \\
\text { más consciente de lo } \\
\text { que siente }\end{array}$ & $\begin{array}{l}\text { Reflejar } \\
\text { sentimientos del que } \\
\text { habla }\end{array}$ & $\begin{array}{l}\text { "Te frustra que } \\
\text { siempre te acuse de } \\
\text { ser el que más habla } \\
\text { en clase" "Te duele } \\
\text { que te acuse de } \\
\text { quitarle el bocadillo" }\end{array}$ \\
\hline Resumir & $\begin{array}{l}\text { Revisar el progreso } \\
\text { que ha habido Juntar } \\
\text { hechos e ideas } \\
\text { importantes }\end{array}$ & $\begin{array}{l}\text { Repetir los hechos y } \\
\text { las ideas principales }\end{array}$ & $\begin{array}{l}\text { "Entonces, si no te he } \\
\text { entendido mal A pegó } \\
\text { a C y a ti te ha } \\
\text { molestado que te vean } \\
\text { como el causante de la } \\
\text { pelea" "Has hablado } \\
\text { de A y B pero no } \\
\text { entiendo qué tiene que } \\
\text { ver C en todo esto" }\end{array}$ \\
\hline
\end{tabular}

Nota: Tomado de Torrego (2003). 
Los beneficios de la escucha activan empática son numerosos; la parte a quien se le devuelve su relato resumido se siente valorada, escuchada, comprendida; la otra puede escuchar, así como detectar la información que no fue capaz de entender cuando la exponía su contrario. También ayuda a quien realizó el relato a entender con claridad sus propios intereses.

\section{CONCLUSIONES}

La experiencia personal, la recopilación de buenas prácticas en centros escolares, así como la consulta de múltiples publicaciones en este campo, llevan a pensar que, aun existiendo modelos claros de mediación y tipologías concretas a nivel escolar, cada centro educativo deberá elegir aquella forma que mejor pueda adaptarse a sus peculiaridades, situación contextual. Incluso, para Vinyamata (2003) "los diversos métodos no pueden considerarse incompatibles, sino que más bien acaba resultando complementario" (p. 13).

En efecto, el interés del medidor por prestar una ayuda eficaz, le conducirá a conciliar métodos, flexibilizando el proceso mediador, alejándolo de posturas que intenten demostrar la superioridad de una metodología u otra. Cada caso, cada situación lo llevará a la utilización de un método u otro, con el fin último de ayudar a quien lo ha solicitado, en el ámbito de la mejora de la resolución de conflictos; pudiendo ayudar de manera importante a generar una cultura de la paz y desarrollar unas competencias sociales, en todos los sectores de la comunidad educativa, que le permitan relacionarse mejor.

Igualmente, el compromiso personal del grupo con el propio centro, aumentará desde la participación activa en la realización de actuaciones encaminadas a traducir, en acciones prácticas los valores, las actitudes, contribuyendo, tanto al crecimiento personal, como al compromiso de los diferentes actores en la cohesión de la comunidad educativa. La incorporación de la mediación escolar como estrategia de resolución de conflictos en un centro educativo, necesitará de compromiso, así como nivel de implicación importante a todos los miembros de la comunidad educativa; comenzando por el equipo directivo, profesores, alumnado, los padres y el personal no docente.

Así mismo el sentar bien las bases antes de crear un grupo de mediadores, será un elemento clave en el éxito de cualquier programa de mediación. Se trata en todo momento de un proyecto a largo plazo, que no sólo debe quedarse en la creación del sistema, sino que deberá buscar 
extender en el centro la cultura de la mediación, resolución pacífica y dialogada de los conflictos. En simples palabras, se puede describir como la intención y voluntad de las personas en resolver una disputa. La mediación eficaz depende de factores tales, como la hostilidad entre las partes, la cantidad de recursos disponibles a distribuir, el poder entre las partes en disputa, entre otras.

Sin embargo, a pesar de la eficacia de las conductas de mediación, se observa la importancia de determinadas técnicas que favorece la creación de un clima que permite suavizar las diferencias entre las partes. Se hace referencia a: la escucha activa empática y la reenmarcación de las frases negativas. La comunicación es un elemento esencial en la resolución de los conflictos, tanto que se puede definir como el proceso de aportar a las partes los recursos comunicativos propios para que puedan solucionarlos.

Durante este proceso, las personas implicadas hablan sobre sus posturas, necesidades, sentimientos, deseos, lanzan reproches, se posicionan ante el problema; los mediadores fomentan la expresión de estos deseos, necesidades, entre otros, de forma constructiva, para que su escucha sirva de ayuda. En consecuencia, la resolución pacífica de conflictos es una habilidad social que contribuye y enriquece la vinculación entre las personas. Desde una posición colaborativa, el proceso de resolución de conflictos implicará:

1.- Consideración de los intereses de la otra parte involucrada en el proceso.,

2.- Dispuesto/a ceder en las posiciones personales para llegar a una salida.

3.- Que beneficie a las partes involucradas en el conflictos.

4.- A fin de mantener, cuidar y enriquecer la relación, si es parte de las expectativas.

Por tanto, el proceso de resolución pacífica de conflictos involucra reconocer igualdad dederechos, oportunidades entre las partes en la búsqueda de solución que satisfaga a ambas, reestablecer la relación posibilitando la reparación si fuere necesario. Por eso, la escuela debe ir más allá para constituirse como un lugar en el que sea posible construir formas alternativas de relacionarse (no violentas), proporcionar ambientes favorables donde proliferen vínculos armoniosos entre los actores sociales que facilite los procesos de aprendizaje, posibilite una educación como desarrollo humano a lo largo de su vida. 


\section{REFERENCIAS}

Arráez, M (2010). Teoría de la Acción comunicativa. Investigación al día; p.9.

Atkinson, J.W. (1964). An introduction to motivation. Princeton, NJ: Van Nostrand. Disponible: psicologiaymente.com > psicologia > teoria-expectativa-valor-atkinson. (Consultado, 2020 Febrero 07).

Cardoso, N. (2014). La comunicación desde una perspectiva de comunicación comunitaria.Disponible:http://www.catedras.fsoc.uba.ar/cardoso/apuntegeneraldecom unitaria.pdf. (Consultado, 2020 Febrero 07).

Cascón, P. (s.f.). Educar en y para el Conflicto. Universidad Autónoma de Barcelona. España.Disponible:dehttp://spip.catedu.es/escuela_de_paz/IMG/pdf/educ._eny_para_c onflicto-110-Gascon.pdf. (Consultado, 2020 Febrero 06).

Cuenca de Ramírez, N (2009). La Negociación, la Mediación y la Conciliación: Técnica de resolución de conflictos para los abogados. Trabajo de grado de no publicación, Universidad Bicentenaria de Aragua. Disponible:www.ucla.edu.ve/viacadem/redine/.../TrabajosTodos/PRevVol1N22011Do c.doc. (Consultado, 2019, Mayo 10)

Chaux, E. (2002). Buscando pistas para prevenir la violencia urbana en Colombia: Conflictos y agresión entre niños[as] y adolescentes en Bogotá. Revista de Estudios Sociales, núm. 12,junio, 2002, pp. 43-53 Universidad de Los Andes Bogotá, Colombia. Disponible:http://www.redalyc.org/articulo.oa?id=81501205. (Consultado, 2020 Febrero 06).

Fernández, I. (2004). Prevención de la violencia y resolución de conflictos. Madrid: Narcea

Fuenmayor, Y. (2012). Comunicación asertiva del gerente y resolución de conflictos en Educación Media general. Tesis de Maestría. Universidad Rafael Urdaneta.

Galtung, J (2003) .Trascender y transformar. Una introducción al trabajo de conflictos, México, Transcend - $\quad$ Quimera. Disponible: https://www.researchgate.net>publication>44813340. Teoría de Conflicto. (Consultado, 2019 Octubre 07).

Griffin, R. (2011). Administración. México: CENGAGE Learning. Décima edición.

Holaday, L. (2002). Stage development theory: A natural framework for understanding the mediation process. NegotiationJournal, 18 (3), p. 191-210.

Lederach, P. El abecé de la paz y los conflictos. Educación para la paz. Madrid: Los Libros de la Catarata, 2000.

Likert, R y Likert, J (1976). New ways of managing conflict. Nueva York: McGrawHill. 
Maturana, H (2002). El sentido de lo humano. Santiago de Chile: Ediciones Pedagógicas Chilenas.

Moreno, M. y Sastre, G. (2010) Conflictos y emociones: un aprendizaje necesario, Facultad de Psicologia, Ciències de 1’Educació i de 1’EsportBlanquerna, UniversitatRaonLlul.

McClelland, D (1965) Teoría de la Motivación de McClelland. Disponible: www.academia.edu > Teoria_de_la_motivación_según_McClelland. (Consultado, 2020 Febrero 06).

Mockus, A. (2002). La Educación para Aprender a Vivir Juntos. Convivencia como Armonización de Ley, Moral y Cultura. PERSPECTIVAS. Revista trimestral deeducación comparada, Volumen (XXXII, n. 1), 19 - 37. Disponible: Recuperado dehttp://www.ibe.unesco.org/fileadmin/user_upload/archive/Publications/Prospects/Pr ospectsPdf/121s/121smock.pdf. (Consultado, 2019, Octubre 30)

Munné, M y Mac-Cragh, P (2006.) Proyecto Armonía: Mejorar la Convivencia Escolar. Consejería de Educación de la Junta de Castilla y León.

Ortega Ruiz R. (2004). Conferencia Construir la Convivencia para prevenir la Violencia. Universidad de Córdova.

Pardo, A. (2014). Conflictos escolares. Sorkari. Atención Integral al desarrollo de la persona. Disponible: http://www.sorkari.com/pdf/Conflictos_Bulling.pdf. (Consultado, 2020 Febrero 05).

Prada, J y López, A (2008) La mediación como estrategia de resolución de conflictos en el ámbito escolar. Disponible: Documentación social, ISSN 0417-8106, Nº 148, 2008, págs. 99-116 Idioma: español. DIALNET MÉTRICAS: 6 Citas. (Consultado, 2020 Febrero 06).

Raposo, S (2012) Principios en los que sustenta la mediación. Disponible: www.camaranavarra.com > Camara > highlight > principios-en-los-qu. (Consultado, 2020 Febrero 06).

Ridao, S (2008) Análisis pragmalingüístico de resoluciones de conflictos: Las mediaciones laborales. Disponible:books.google.co.ve > books. (Consultado, 2020 Febrero 04).

Rodríguez, R. (2007). Los Planes de Convivencia como herramientas para prevenir los conflictos escolares. Murcia: Ponencia Jornadas Europeas Sobre Convivencia Escolar.

Rozenblum, S. (1998). Mediación en la escuela. Buenos Aires: Aique.

Shapiro, D. (2002). Negotiating emotions. Conflict Resolution Quarterly, 20 (1),

p. $67-82$ 
Torrego, C. (2003). Mediación de conflictos en instituciones educativas. Manual para la formación de formadores. Madrid: Narcea.

Viana, M (2015) La Mediación: características, modelos, proceso, técnicas y herramientas de la persona mediadora, y límites a la mediación, sitio web 292. Disponible:http://La\%20Mediaci\%C3\%B3n,\%20caracter\%C3\%ADsticas,\%20odelos, $\% 20$ proceso,\%20t\%C3\%A9cnicas\%20o\%20herramientas\%20de\%20la\%. (Consultado, 2020 Febrero 04).

Viñas, J. (2004): Conflictos en los centros educativos. Cultura organizativa y mediación para la convivencia, Barcelona, Graó. 